\title{
Differential metabolism-associated gene expression of duck pancreatic cells in response to two subtypes of duck hepatitis A virus type 1
}

Shaohua SHI

Pourlty disease

Zhen CHEN

Poultry disease

Yu HUANG ( $\square$ huangyu_815@163.com )

Cuiqin HUANG

Poultry disease

Rongchang LIU

Poultry disease

Longfei CHENG

Poultry disease

Guanghua FU

Poultry disease

Hongmei CHEN

Poultry disease

Chunhe WAN

Poultry disease

Qiuling FU

Poultry disease

\section{Research article}

Keywords: Duck hepatitis A virus type 1, Duck pancreas, Metabolism disorder

Posted Date: February 28th, 2020

DOI: https://doi.org/10.21203/rs.2.24829/v1

License: (a) (i) This work is licensed under a Creative Commons Attribution 4.0 International License.

Read Full License 


\section{Abstract}

Background: Duck hepatitis A virus type 1 (DHAV-1) causes a highly contagious disease in domestic ducklings, which is traditionally characterized by lesions in the liver and rarely in the pancreas. However, several outbreaks of DHAV-1, which were characterized by yellow coloration and hemorrhage in pancreatic tissues, have occurred in China. Genomic sequencing indicated the presence in the variation rates of 3.4-6.5\% in the genome of the novel DHAV-1 compared with DHAV-1. The antigenic relationship between the novel DHAV-1 and DHAV-1 indicated large variation. So the novel DHAV-1 was named as DHAV-1 subtype (DHAV-1a). But the mechanism involved in infection of DHAV-1a is still unclear. Results: In the present study, transcriptome analysis of duck pancreas infected with DHAV-1 and DHAV-1a was carried out. Following deep sequencing with Illumina-Solexa, a total of $53.9 \mathrm{~Gb}$ clean data were obtained from the cDNA library of the pancreas and a total of 29,597 unigenes with an average length of 993.43 bp were generated following de novo sequence assembly. The expression levels of D-3phosphoglyceratedehydrogenase, phosphoserine aminotransferase, phosphoserine phosphatase, which are involved in the glycine, serine and threonine metabolism pathway, were significantly down-regulated in the DHAV-1a-infected group compared with those of DHAV-1-infected group. Conclusion: These findings offered different expression levels of metabolism-associated genes between the novel DHAV$1 \mathrm{a}$ and DHAV-1, indicating that intensive metabolism disorder may contribute to different phenotypes of DHAV-1-infection.

\section{Background}

Duck viral hepatitis (DVH) is a highly fatal contagious disease of ducklings, characterized by clinical sign of opisthotono and by liver hemorrhagic lesion. This causes mortality rates of approximately $95 \%$ in ducklings within a week. The causative agents of DVH include duck hepatitis virus type 1 (DHV-1), DHV-2 and DHV-3. Recently, DHV-1 along with two other types was classified as the species Avihepatovirus A of the genus Avihepatovirus in the family Picornaviridae and was renamed as duck hepatitis A virus (DHAV) [1]. DHAV is divided into three serotypes as follows: DHAV-1, which is the classical type and is the most widespread strain, followed by DHAV-2 and DHAV-3.

To the best of our knowledge, DHAV-1 is distributed worldwide and DHAV-2 has been isolated only in Taiwan till date [2-3]. DHAV-3 was mainly reported in Korea and China [4-7]. Generally, the lesion in the liver is a typical lesion of DVH caused by DHAV-1. However, Guérin et al. reported that several DHVinfections of young Muscovy ducklings exhibited nervous signs and pancreatitis in 2005 [8]. Several outbreaks of DHAV-1, which were characterized by yellow coloration and hemorrhage in pancreatic tissues, have occurred in China in 2011 [9]. Genomic sequencing indicated the presence in the variation rates of 3.4\%-6.5\% in the genome of the novel DHAV-1 compared with DHAV-1 [10]. The antigenic relationship between the novel DHAV-1 and classical type DHAV-1 indicated large variation. The novel DHAV-1 was then named as duck hepatitis A virus type 1 subtype (DHAV-1a) [11]. The mechanism involved in infection of DHAV-1a is still unclear. The host response to the infection of DHAV-1a requires experimental validation. 
The host-pathogen interaction is largely affected by the virulence of the pathogen and the host immune response [12]. However, several aspects of DHAV-1a-host interactions remain to be clarified.

Transcriptomics is the analysis of genome-wide RNA expression and is an available approach to characterize host and pathogen processes in infectious diseases. This method also provides a clear understanding of the host defense mechanisms during the viral infection. To date, the data on the transcriptome profile of the duck pancreatic tissue and its response to viral infection at the transcriptome level are lacking. Moreover, the differences of host responses to DHAV-1 and DHAV-1a have not been reported to date.

In the present study, transcriptome sequencing was applied to determine the gene transcriptome pattern of duck pancreatic tissues infected with DHAV-1 and DHAV-1a. Dramatic differences were observed in the genes involved in the glycine, serine and threonine metabolism pathway, indicating that intensive metabolism disorders may contribute to the different phenotypes of DHAV-1-infection.

\section{Results}

\section{Gross lesions of DHAV-1 and DHAV-1a-infected ducklings}

The infection experiments were conducted with DHAV-1a GD1206 and DHAV-1 FZ86 strains at the dose of $10^{5.0}$ ELD50 per ducks. The ducks infected with DHAV-1 FZ86 strain indicated clinical symptoms of depression, lethargy, and opisthotonos. The major lesions that were typically enlarged with petechial and ecchymotic hemorrhages in the liver and kidney were observed during necropsy. No visible lesions were noted in the pancreas. In contrast to these observations, the ducklings infected with DHAV-1a GD1206 strain demonstrated no typical signs during the course of infection. The major affected organ in the DHAV-1a group was the pancreas which exhibited lesions of yellowing or hemorrhage. No hemorrhagic change was noted in the liver of the DHAV-1a-infected ducklings. No gross lesions were observed in the mock-infected control group. The pancreatic samples were DHAV-1- or DHAV-1a-positive in the DHAV-1- or DHAV-1a-group as determined by RT-PCR.

\section{RNA-seq and assembly of transcriptome data from the pancreas of the ducklings}

Following Illumina-Solexa deep sequencing, a total of $183 \mathrm{M}$ raw reads were obtained in the cDNA library derived from pancreatic tissues. The removal of low-quality reads (i.e., reads containing only adaptors and empty reads) resulted in the identification of clean reads with total residues of $53.9 \mathrm{~Gb}$ clean data. Following de novo sequence assembly, a total of 29,597 unigenes with an average length of $993.43 \mathrm{bp}$ were generated. BLAST and ORF analyses indicated that only 9,387 targets matched the known genes among the total number of unigenes. The sequencing data from the GD1206- and FZ86-infected groups were submitted to the NCBI database (accession number: SRR7239978, SRR7239979, SRR7239984, SRR7239985, SRR7239988, and SRR7239989).

The heatmaps indicated the top differentially expressed genes (DEGs) and the classification of gene expression profiles in different subtype DHAV-1-infection (Figures 1). In order to obtain a global view of 
the change in duck gene expression among different experimental groups, three paired comparisons (DHAV-1 vs. control, DHAV-1a vs. control, DHAV-1a vs. DHAV-1) were performed. RNA-seq analysis detected 3,340 and 5,919 genes, which were expressed at significantly different levels in DHAV-1- and DHAV-1a-infected animals, respectively compared with those noted in the control group $(P<0.05)$. DHAV-1 infection contributed to the differential expression of 2,031 genes that were up-regulated and 1,309 genes that were down-regulated in the pancreatic tissues of the infected animals compared with those noted in the control ducklings. Moreover, 3,308 genes were up-regulated and 2,611 genes were down-regulated in the DHAV-1a-infection animals. A total of 1,913 genes were differentially expressed in DHAV-1a-infected animals compared with those of the DHAV-1-group. Specifically, 834 genes were down-regulated and 1,079 genes were up-regulated (Figure 2).

\section{Function analysis of the transcriptome data derived from the pancreatic tissues of different type DHAV- infected animals}

We performed GO enrichment analysis by functional annotation clustering of DEGs and annotated DEGs into three groups, including biological processes (BP), cellular components (CC), and molecular function (MF).

The putative functions of the unigenes in the libraries derived from DHAV-infected ducklings were analyzed using GO. The analysis of the GO categories of the DHAV-1-group indicated that the differentially expressed genes were mapped to 61 categories of BP, CC and MF (Figure 3A). The category of $\mathrm{BP}$ included mainly the genes involved in the positive regulation of interleukin-12 production, myeloid leukocyte activation and $\mathrm{T}$ cell proliferation. The majority of the corresponding genes in CC category were involved in the external side of plasma membrane, the oligosaccharyltransferase complex and the integral component of the membrane. The category of MF included genes that were involved in the nonmembrane spanning protein tyrosine kinase activity, antioxidant activity and calcium-dependent phospholipid binding.

The putative functions of the unigenes in the pancreatic libraries of the DHAV-1a-infection were analyzed using GO. The analysis of the GO categories indicated that the differentially expressed genes were mapped to 61 categories including BP, CC and MF (Figure 3B). The majority of the corresponding genes in the BP category were involved in cholesterol efflux, positive regulation of cell cycle and translation. The category of the $\mathrm{CC}$ included genes mainly involved in the cytosolic large ribosomal subunit, extracellular space and ribosome. The majority of the genes of the MF category included genes involved in the structural constituent of ribosome, cytokine activity and NADH dehydrogenase (ubiquinone) activity.

The putative functions of the unigenes in the pancreatic libraries of DHAV-1a-infected ducklings were compared with those of the DHAV-1-infected ducklings and were analyzed using GO. The analysis of the GO categories indicated mapping of differentially expressed genes to 61 categories of BP, CC and MF (Figure $3 \mathrm{C}$ ). The BP category included mainly genes that were involved in the oxidation-reduction process, the negative regulation of apoptotic process and the myeloid leukocyte activation. The CC category included mainly genes that were involved in the extracellular space, protein-extracellular matrix and 
ribosome. Most of the corresponding genes in the MF category were involved in the antioxidant activity, G-protein coupled peptide receptor activity and structural constituent of ribosome.

\section{Pathway analysis of DEGs based on KEGG following different DHAV-1-infection}

The KEGG database was used to analyze specific pathways in order to further define DEG function in the duckling pancreatic tissue following different DHAV-1-infections. The top 20 enrichment KEGG pathways were listed in Figure 4 according to their Q-value $<0.05$ (Table 1).

A total of 6 functional categories were identified that played important roles in the DHAV-1 FZ86- and DHAV-1a GD1206-mediated infections. These categories were mainly classified into immune system categories, including the Toll-like receptor signaling pathway. However, significant KEGG enrichment in the DHAV-1a group was also involved in the metabolism function, including the glycine, serine and threonine metabolism pathway.

\section{Verification of DEG identification by real-time RT-PCR}

In order to verify the differential gene expression levels obtained from the transcriptome sequencing data, we analyzed the expression levels of 10 genes of immune and metabolism-associated genes that were mainly involved in host immune defense responses and metabolism in the two phenotype DHAV-1 infection groups. The genes examined as following: GNMT-like, GCAT, CBS, PHGDH, SERCA, PLCY, TLR2, TLR4, TLR7 and IFNa, were significantly differently expressed compared with the control ones $(P<0.05)$, indicating the reliability of the transcriptome sequencing data (Table 2).

\section{Discussion}

Duck virus hepatitis is mainly caused by DHAV-1 and is a malignant disease in ducklings, which affects severely the duck industry worldwide. Previous studies performed on DHAV have focused only on the pathogenicity and the host response to DHAV-1 infection. The comparative pathogenicity of DHAV-1 and DHAV-1a strains in ducklings of different species has been well documented [13-14]. However, the different mechanisms between DHAV-1 and DHAV-1a strains remain unknown.

In the present study, the ducklings that were inoculated with DHAV-1 FZ86 indicated massive haemorrhages on the liver surface. The ducklings that were inoculated with the DHAV-1a GD1206 strain exhibited loss of appetite, lying prone, diarrhea and depression. The gross lesion of the yellowed or hemorrhagic pancreatitis was observed in DHAV-1a infected ducklings. However, no consistent nervous disorders were observed in the present study, which was not in line with the observation by Guérin et al. [8]. Cha et al. described that a DHAV-3 strain induced only liver discoloration without hemorrhagic mottling, lymphocyte infiltration and bile duct hyperplasia as determined by histology of the lesions [15]. These studies revealed the diversity in the pathogenicity of DHAV.

Transcriptome analysis is a promising tool that can provide a comprehensive understanding of the molecular mechanisms involved in specific biological processes and diseases [16]. Previous studies have 
described the transcriptome profile of DHAV-, Reovirus- and DHBV-infection in ducks [17-20]. In the present study, transcriptome sequencing was employed to explore and compare the gene expression patterns of infection with different subtype DHAV-1 in duck pancreatic tissues, with the aim to compare different molecular events during DHAV-1 and DHAV-1a infection.

Following analysis of the clean reads via BLAST and ORF, a total of 9,387 matched known genes were obtained. The DEGs were annotated and categorized by GO and KEGG signaling pathway analyses, which demonstrated that the majority of these genes in DHAV-1 and DHAV-1a infection groups were classified into immune system and metabolism categories. The investigation of the transcriptome of DHAV-1-infected and the control ducklings indicated that DHAV-1 infection caused down-regulation of the expression of genes associated with metabolic pathways and inhibition of the metabolism of the host cell. In addition, up-regulation of the expression levels of the immune-associated genes was noted that resulted in inhibition of viral replication and progression of viral infection [21]. Similarly, the up-regulation of the expression of immune genes and the down-regulation of certain metabolism-related genes were observed in DHAV-1a infection group compared with the control in the present study. However, significant KEGG enrichment was noted in the DHAV-1a infection group compared with DHAV-1 infection group that was mainly involved the metabolism function, including the glycine, serine and threonine metabolism pathway. The result suggested that the differences in the metabolism function of the DHAV-infection group may contribute to the different phenotypes of DHAV-1.

D-3-phosphoglycerate dehydrogenase (PHGDH), phosphoserine aminotransferase区PSAT囚and phosphoserinephosphatase (PSP) are involved in the three-enzyme serine biosynthesis [22]. Initially, 3phosphoglycerate is converted to 3-phosphohydroxypyruvate by the enzyme PGDH. Subsequently, PSAT converted 3-phosphohydroxypyruvate to 3-phosphoserine. Finally, 3-phosphoserine was converted into Lserine by PSP [23]. L-serine is an important precursor involved in various processes, such as synthesis of proteins and phospholipids as well as the synthesis of tetrahydrofolate metabolites and specific amino acids, namely glycine, cysteine, D-serine [24-25]. Previous studies have shown that L-serine supplementation can attenuate alcoholic fatty liver formation in mice and rats [26]. In the present study, the expression levels of the PHGDH, PSAT and PSP genes were significantly down-regulated in the DHAV1a-infected ducklings compared with those noted in DHAV-1 group, indicating that serine metabolism disorders were involved in DHAV-1a-infection.

L-serine dehydratase $(\mathrm{SDH})$ catalyzes the deamination of L-serine to yield ammonia and pyruvate. This enzyme uses L-threonine as a substrate to yield 2-oxobutanoate, which is a part of the valine, leucine and isoleucine biosynthetic pathway. The down-regulation of serine dehydratase levels suggested that the pathway of serine to pyruvate was impaired and further affected the valine, leucine and isoleucine biosynthetic pathway [27-29].

The innate immune system is essential for recognizing pathogen infection and for the establishment of effective host defense. During the process of innate immune response, highly conserved pathogenassociated molecular patterns (PAMP) are detected by pattern recognition receptors (PRR) [30]. Three 
classes of PRRs have been shown to be involved in the recognition of virus-specific components in innate immune cells, namely Toll-like receptors (TLRs), retinoic acid-inducible gene I (RIG-I)-like receptors (RLRs), and nucleotide oligomerization domain (NOD)-like receptors s (NLRs) [31]. In the present study, almost similar innate immune responses were observed in both subtypes from DHAV-1 infected animals.

TLRs play a critical role in innate immune responses. In recent years, the role of innate immunity and its interaction with adaptive immunity has been mainly explored. In the TLR pathway, TLR2 plays a critical role in the induction of innate and inflammatory responses [32-33]. Although TLR2 recognizes various bacterial components, recent studies have indicated that TLR2 is triggered by the HCV protein core and NS3 leading to the activation of inflammatory cells [34-35]. In the present study, TLR2 gene was 10.63- or 7.62-fold higher than that of the control group ducklings in DHAV-1a/DHAV-1 infection respectively, indicating that TLR2 may be involved in the host response to DHAV infection. TLR4 and CD14 have recently been shown to be the major lipopolysaccharide (LPS) receptors. Mutations in mouse and human TLR4 were found to be associated with hyporesponsiveness to LPS and confer an increased risk of gramnegative infections [36-38]. In addition to the interaction with LPS, the TLR4/CD14 complex interacts with other viruses and proteins, such as the respiratory syncytial virus and fibrinogen [39-41]. In the present study, TLR4/CD14 expression in DHAV-1a and DHAV-1 infection were highly up-regulated compared with that of non-infected ducklings, indicating that TLR4/CD14 may be involved in the host response to DHAV1a and DHAV-1 infection. Surprisingly, TLR4 expression was significantly higher with an increase of 51.63- and 38.59-fold higher in DHAV-1a and DHAV-1 groups respectively compared with the mocked group. TLR7 can detect single stranded RNA (ssRNA) molecules and induces pro-inflammatory factors, such as the type I interferon to stimulate the body's non-specific immune response. Activation of TLR7 initiates downstream signaling cascades via transcription factors, such as IRF7. This induces the production of pro-inflammatory cytokines and chemokines that are involved in various viral infection outcomes, including spontaneous clearance and viral persistence [42]. The potential implication of TLR7 in the innate immune response against hepatitis $C$ virus was postulated [43-44]. Nazmi et al. suggested that the TLR7 response following Japanese encephalitis virus infection promoted type- 1 interferon production and generation of an antiviral state, contributing to the protective effect of systemic infection [45]. In the present study, the up-regulation of the expression levels of TLR7, IRF7 and IFN- $\beta$ was noted in DHAV-1a and DHAV-1 group, which was consistent with the findings described in previous studies [45-46].

\section{Conclusions}

In the present study $₫$ transcriptome analysis of pancreatic tissues derived from DHAV-1 and/or DHAV-1ainfected ducks was reported. The infection of DHAV-1 a caused yellowing and hemorrhagic lesions in the pancreatic tissues of the ducklings and was associated with differences in the expression levels of D-3phosphoglyceratedehydrogenase, phosphoserine aminotransferase, phosphoserine phosphatase, which are involved in the glycine, serine and threonine metabolism pathway. The expression levels of the aforementioned genes were significantly down-regulated in the DHAV-1a-infected group compared with those from DHAV-1-infected group, indicating that intensive metabolism disorder may contribute to the different phenotypes of DHAV-1 infection. 


\section{Methods}

\section{Animal Experiment}

One-day-old male Muscovy ducklings (Cairina moschata) were purchased from a commercial hatchery farm. Each duckling has free access to food and water and shared the same environmental conditions at $35^{\circ} \mathrm{C}$. The temperature dropped $1{ }^{\circ} \mathrm{C}$ every day. The light was continuously available during the animal experiments. Serum samples and cloacal swabs were collected from the ducklings prior to viral challenge. The presence of antibody and antigen was determined using indirect enzyme linked immunosorbent assay (ELISA) and reverse transcription polymerase chain reaction (RT-PCR) methods, respectively in order to confirm the DHAV-1 and DHAV-1 a negative statuses. Four days later, sixty of 5day-old healthy Muscovy ducklings, which were negative for DHAV-1 and DHAV-1a antigens and antibodies, were randomly divided into three groups ( $n=20$ for each group). The ducklings in each group were challenged intramuscularly with $10^{5.0}$ ELD50 (50\% egg lethal dose) DHAV-1 FZ86 strain and DHAV1a GD1206 strain in $200 \mu \mathrm{l}$ phosphate buffer saline (PBS) or mock-infected with PBS. Each group of ducklings was housed in separate isolators (Suzhou Fung's laboratory Animal Equipment Co., Ltd.) with free access to food and water throughout the experimental period. Each group of ducklings shared the same environmental conditions at $31^{\circ} \mathrm{C}$. The temperature dropped $1{ }^{\circ} \mathrm{C}$ every day until to $25^{\circ} \mathrm{C}$. The light was continuously available during the animal experiments. Daily clinical symptoms were monitored.

At $24 \mathrm{~h}$ post-inoculation, six ducklings in each group were randomly selected. The ducklings were administrated intravenously with sodium pentobarbital ( $40 \mathrm{mg} / \mathrm{kg}$ body weight; Sigma-Aldrich, USA) and then humanly sacrificed in narcosis [47]. The pancreatic samples were harvested and pooled into two tubes ( 3 samples/tube, $0.5 \mathrm{~g}$ each sample) for transcription analysis. Concomitantly, the pancreatic samples were also detected by RT-PCR in order to confirm DHAV-1- or DHAV-1a-infection. The remained fourteen ducklings of each group were observed for three days. At the end of the observed period, the ducklings were administrated intravenously with sodium pentobarbital $(40 \mathrm{mg} / \mathrm{kg}$ body weight; SigmaAldrich, USA) and then humanly sacrificed in narcosis [47]. The gross lesions were observed.

\section{Host gene expression associated with DHAV-1a infection}

\section{RNA extraction, library construction, and sequencing}

Total RNA was extracted using RNAiso Plus Total RNA extraction reagent (TaKaRa, Shiga, Japan) according to the manual instructions. RNA degradation and contamination was monitored on $1.0 \%$ agarose gels. RNA purity was confirmed using the NanoPhotometer ${ }^{\circledR}$ spectrophotometer (IMPLEN, CA, USA). RNA concentration was measured using the Qubit ${ }^{\circledR}$ RNA Assay Kit in Qubit $\circledast 2.0$ Fluorometer (Life Technologies, CA, USA). RNA integrity was assessed using the RNA Nano 6000 Assay Kit of the Agilent Bioanalyzer 2100 system (Agilent Technologies, CA, USA) and subsequently employed as a template for the synthesis of double-stranded CDNAs. Sequencing libraries were generated using NEBNext ${ }^{\circledR} U$ Ultra $^{\text {TM }}$ RNA Library Prep Kit for Illumina ${ }^{\circledR}$ (NEB, CA, USA) following the manufacturer's recommendations. Finally, index codes were added to the attribute sequences of each sample. The clustering of the index-coded 
samples was performed on a cBot Cluster Generation System using TruSeq PE Cluster Kit v3-cBot-HS (Illumina) according to the manufacturer's instructions. Following cluster generation, the library preparations were sequenced on an Illumina Hiseq 4000 platform and paired-end reads were generated. The RNA-seq data from this study were submitted to the NCBI Sequence Read Archive (SRA) (http://www.ncbi.nlm.nih.gov/sra/).

\section{Transcriptome data analysis}

Differential expression analysis of the three groups was performed using the DESeq R package (1.10.1). DESeq provided statistical routines for determining differential expression in digital gene expression data using a model based on the negative binomial distribution. The resulting P-values were adjusted using the Benjamini and Hochberg's approach for controlling the false discovery rate. The genes with an adjusted P-value of $<0.05$ found using DESeq were assigned as differentially expressed.

\section{GO enrichment analysis and KEGG pathway enrichment analysis}

Gene Ontology (GO) enrichment analysis of the differentially expressed genes (DEGs) was implemented by the topGO R packages based Kolmogorov-Smirnov test. The GO terms with the corrected $P$ value less than 0.05 were considered significantly enriched in the differential expressed genes.

Kyoto Encyclopedia of Genes and Genomes (KEGG) is a database resource for understanding high-level functions and utilities of the biological systems, such as the cell, organism and the ecosystem. Specific molecular-level information is collected notably from large-scale molecular datasets generated by genome sequencing and other high-throughput experimental technologies (http://www.genome.jp/kegg/) [48]. The KOBAS software was used to assess the statistical enrichment of differentially expressed genes in the KEGG pathways [49]. The KEGG terms with a corrected P-value (Q-value) less than 0.05 were considered significantly enriched in the differential expressed genes.

\section{Verification by real-time RT-PCR}

The expression levels of the differentially expressed genes were also assessed by real-time RT-PCR in order to confirm the sequencing data. The individual primer sequences of the 10 target genes and of the internal reference gene (GAPDH) [50] were listed in Table 3. Following reverse transcription, a SYBRßFast qPCR Mix (Takara, Beijing) and a Mastercycler ep realplex (eppendorf) were used according to the manufacturers' instructions. The reaction conditions were as follows: $95^{\circ} \mathrm{C}$ for $1 \mathrm{~min}$, followed by 40 cycles of $95^{\circ} \mathrm{C}$ for $10 \mathrm{sec}$ and $60^{\circ} \mathrm{C}$ for $40 \mathrm{sec}$. A final solubility curve analysis was completed. The relative mRNA levels were compared to those of the mock-infected ducks and the mean values were calculated using the threshold cycle $2^{-\triangle \Delta C T}$ method [51]. The comparisons of the experimental groups were estimated by the Student's $t$-test with a two-tailed analysis in order to determine significant differences. P-values less than 0.05 were considered significant.

\section{Abbreviations}


KEGG: Kyoto encyclopedia of genes and genomes; GO: Gene ontology; RT-PCR: Reverse transcription polymerase chain reaction; ELISA: Enzyme linked immunosorbent assay; DEG: Differentially expressed genes; BLAST: Basic local alignment search tool囚ORF: Open reading frame.

\section{Declarations}

\section{Ethics Approval and Consent to Participate}

All animal experiments were approved by the animal welfare committee of Fujian Academy of Agricultural Sciences (No.1306175FAAS) and complied with the guidelines of Fujian laboratory animal welfare and Fujian Administrative Ethics Committee of Laboratory Animals.

\section{Consent for publication}

Not applicable.

\section{Availability of data and materials}

The raw data has been deposited in NCBI database, with [DHAV-1-infected group accession number》 SRR7239978 and SRR7239979], [Control group accession numberखSRR7239984 and SRR7239985], and [DHAV-1a-infected group accession numberखSRR7239988 and SRR7239989],

[https://www.ncbi.nlm.nih.gov/sra/?term=SRR7239978,

https://www.ncbi.nlm.nih.gov/sra/?term=SRR7239979,

https://www.ncbi.nlm.nih.gov/sra/?term=SRR7239984,

https://www.ncbi.nlm.nih.gov/sra/?term=SRR7239985,

https://www.ncbi.nlm.nih.gov/sra/?term=SRR7239988,

https://www.ncbi.nlm.nih.gov/sra/?term=SRR7239989]

\section{Competing interests}

The authors declared no conflict of interest.

\section{Funding}

This research was supported by National Key Research and Development Program of China (No. 2017YFD0500802), National Natural Science Foundation of China (No. 31472222), Fund for Modern Agro-industry Technology Research System (No. CARS-42) from the Ministry of Agriculture of P. R. China, Natural Science Foundation of Fujian Province (No. 2017J01060) and Financial Special of Fujian 
Province (No. FJFS-2017). The funders had no role in study design, data collection and analysis, decision to publish, or the manuscript

preparation.

\section{Author's contribution}

SS, $\mathrm{CZ}$ and $\mathrm{HY}$ conceived and designed the experiments; SS and $\mathrm{CZ}$ wrote the original draft preparation; $\mathrm{SS}, \mathrm{CZ}, \mathrm{HC}, \mathrm{LR}, \mathrm{CL}$ and FG performed the experiments; $\mathrm{CH}, \mathrm{WC}$ and $\mathrm{FQ}$ analyzed the data. All authors read and approved the final manuscript.

\section{Acknowledgements}

The authors would like to thank Miss Yajing XU, MedSci, for editing the manuscript.

\section{Authors' Information}

Institute of Animal Husbandry and Veterinary Medicine, Fujian Academy of Agricultural Sciences, Fuzhou, Fujian, China; Fujian Animal Diseases Control Technology Development Center; Fujian Provincial Key Laboratory for Avian Diseases Control and Prevention, Fuzhou, Fujian, China (Shaohua SHIखZhen CHEN囚 Yu HUANG『Longfei CHENG『Guanghua FU『Hongmei CHEN『Chunhe WAN『Qiuling FU『

College of Life Sciences, Longyan University, Longyan, Fujian, China囚Cuiqin HUANG囚

\section{References}

1. Kim M, Kim M, Kwon Y, Lindberg A, Joh S, Kwon H, et al. Development of duck hepatitis A virus type 3 vaccine and its use to protect ducklings against infections. Vaccine. 2009; 27(48): 6688-94.

2. Tseng $\mathrm{C}$, Knowles N, Tsai H. Molecular analysis of duck hepatitis virus type 1 indicates that it should be assigned to a new genus. Virus Res. 2007; 123(2): 190-203.

3. Tseng C, Tsai H. Molecular characterization of a new serotype of duck hepatitis virus. Virus Res. 2007; 126(1-2): 19-31.

4. Fu Y, Pan M, Wang $X, X u$ Y, Yang H, Zhang D. Molecular detection and typing of duck hepatitis A virus directly from clinical specimens. Vet Microbiol. 2008; 131(3-4): 247-57.

5. Kim M, Kwon Y, Joh S, Kim S, Tolf C, Kim J, et al. Recent Korean isolates of duck hepatitis virus reveal the presence of a new geno- and serotype when compared to duck hepatitis virus type 1 type strains. Arch Virol. 2007; 152(11): 2059-72.

6. Liu M, Fanyi M, Li X, Zhang Z, Liu S, Zhang Y. Goose haemorrhagic hepatitis caused by a new subtype duck hepatitis type 1 virus. Vet Microbiol. 2011; 152(3-4): 280-3.

7. Wei C, Su S, Huang Z, Zhu W, Chen J, Zhao F, et al. Complete genome sequence of a novel duck hepatitis A virus discovered in southern China. J Virol. 2012; 86(18): 10247. 
8. Guérin J, Noutary V, Boissieu C, Albaric 0 , Wyers M. Viral pancreatitis and encephalitis of Muscovy ducklings. Vet Rec. 2005; 157(11): 328.

9. Fu G, Chen H, Huang Y, Shi S. Identification and sequence analysis of duck hepatitis A virus type I isolated from Muscovy duckling with pancreatitis. FJ J Agri Sci. 2012; 27(9): 43-5.

10. Chen Z, Fu Q, Chen H, Fu G. Different pathogenicity of duck Hepatitis A virus 1 between pancreatitistype and classical duckling. FJ J Agri Sci. 2013; 28 (10): 939-42.

11. Fu Q, Chen Z, Huang Y, Fu G. Identification of subtype of duck hepatitis A virus type 1 causing pancreatitis. Chin J Vet Med. 2015; 51(7): 36-8.

12. Schneider R, Shenk T. Impact of virus infection on host cell protein synthesis. Annu Rev Biochem. 1987; 56: 317-32.

13. Liu R, Shi S, Huang Y, Chen Z, Chen C, Cheng L, et al. Comparative pathogenicity of different subtypes of duck hepatitis A virus in Pekin ducklings. Vet Microbiol. 2019; 228: 181-7.

14. Zhu T, Qi B, Liu R, Jiang X, Lu R, Xiao L, et al. Comparative pathogenicity of two subtypes (hepatitistype and pancreatitis-type) of duck hepatitis A virus type 1 in experimentally infected Muscovy ducklings. Avian Pathol. 2019; 48(4): 352-61.

15. Cha S, Roh J, Kang M, Kim B, Jang H. Isolation and characterization of a low pathogenic duck hepatitis A virus 3 from South Korea. Vet Microbiol. 2013; 162(1): 254-8.

16. Qian X, Ba Y, Zhuang Q, Zhong G. RNA-Seq technology and its application in fish transcriptomics. OMICS. 2014; 18(2): 98-110.

17. Wang Q, Liu M, Xu L, Wu Y, Huang Y. Transcriptome analysis reveals the molecular mechanism of hepatic fat metabolism disorder caused by Muscovy duck reovirus infection. Avian Pathol. 2018; 47(2): 127-39.

18. Wang X, Zhang J, Meng R, Jiang Y, Liang S, Zhang Y, et al. Host differences affecting resistance and susceptibility of the second generation of a Pekin duck flock to duck hepatitis A virus genotype 3 . Front Microbiol. 2017; 8: 1128.

19. Yan L, Qu S, Liu G, Liu L, Yu Y, Ding G, et al. Comparative transcriptomic analysis of primary duck hepatocytes provides insight into differential susceptibility to DHBV infection. PLoS One. 2016; 11(2): e0149702.

20. Zhang X, Cao C, Liu Y, Qi H, Zhang W, Hao C, et al. Comparative liver transcriptome analysis in ducklings infected with duck hepatitis A virus 3 (DHAV-3) at 12 and 48 hours post-infection through RNA-seq. Vet Res. 2018; 49(1): 52.

21. Zhang Q. Research on the transcriptome of DHAV-1 infected duckling's livers through RNA-Seq. 2013, Huazhong Agricultural University: Wuhan.

22. Hart C, Race V, Achouri Y, Wiame E, Sharrard M, Olpin S, et al. Phosphoserine aminotransferase deficiency: a novel disorder of the serine biosynthesis pathway. Am J Hum Genet. 2007; 80(5): 931-7.

23. El-Hattab A. Serine biosynthesis and transport defects. Mol Genet Metab. 2016; 118(3): 153-9. 
24. Zaki M, Thoenes M, Kawalia A, Nurnberg P, Kaiser R, Heller R, et al. Recurrent and prolonged infections in a child with a homozygous IFIH1 nonsense mutation. Front Genet. 2017; 8: 130.

25. Jiang H, Yu Y, Liu S, Zhu M, Dong X, Wu J, et al. Proteomic study of a Parkinson's disease model of undifferentiated SH-SY5Y cells induced by a proteasome inhibitor. Int J Med Sci. 2019; 16(1): 84-92.

26. Sim W, Yin H, Choi H, Choi Y, Kwak H, Kim S, et al. L-serine supplementation attenuates alcoholic fatty liver by enhancing homocysteine metabolism in mice and rats. J Nutr. 2015; 145(2): 260-7.

27. Sun L, Bartlam M, Liu Y, Pang H, Rao Z. Crystal structure of the pyridoxal-5'-phosphate-dependent serine dehydratase from human liver. Protein Sci. 2005; 14(3): 791-8.

28. Talukdar G, Inoue R, Yoshida T, Ishimoto T, Yaku K, Nakagawa T, et al. Novel role of serine racemase in anti-apoptosis and metabolism. Biochim Biophys Acta Gen Subj. 2017; 1861(1): 3378-87.

29. Ogawa H, Gomi T, Fujioka M. Serine hydroxymethyltransferase and threonine aldolase: are they identical? Int J Biochem Cell Biol. 2000; 32(3): 289-301.

30. Cario E. Bacterial interactions with cells of the intestinal mucosa: Toll-like receptors and NOD2. Gut. 2005; 54(8): 1182-93.

31. Takeuchi O, Akira S. Innate immunity to virus infection. Immunol Rev. 2009; 227(1): 75-86.

32. Aderem A, Ulevitch R. Toll-like receptors in the induction of the innate immune response. Nature. 2000; 406(6797): 782-7.

33. Medzhitov R, Preston-Hurlburt P, Janeway C. A human homologue of the Drosophila Toll protein signals activation of adaptive immunity. Nature. 1997; 388(6640): 394-7.

34. Dolganiuc A, Oak S, Kodys K, Golenbock D, Finberg R, Kurt-Jones E, et al. Hepatitis C core and nonstructural 3 proteins trigger toll-like receptor 2-mediated pathways and inflammatory activation. Gastroenterology. 2004; 127(5): 1513-24.

35. Feldmann G, Nischalke H, Nattermann J, Banas B, Berg T, Teschendorf C, et al. Induction of interleukin- 6 by hepatitis $C$ virus core protein in hepatitis $\mathrm{C}$-associated mixed cryoglobulinemia and $\mathrm{B}$ cell non-Hodgkin's lymphoma. Clin Cancer Res. 2006; 12(15): 4491-8.

36. Arbour N, Lorenz E, Schutte B, Zabner J, Kline J, Jones M, et al. TLR4 mutations are associated with endotoxin hyporesponsiveness in humans. Nat Genet. 2000; 25(2): 187-91.

37. Lorenz E, Mira J, Frees K, Schwartz D. Relevance of mutations in the TLR4 receptor in patients with gram-negative septic shock. Arch Intern Med. 2002; 162(9): 1028-32.

38. Qureshi S, Lariviere L, Leveque G, Clermont S, Moore K, Gros P, et al. Endotoxin-tolerant mice have mutations in Toll-like receptor 4 (TIr4). J Exp Med. 1999; 189(4): 615-25.

39. Kurt-Jones E, Popova L, Kwinn L, Haynes L, Jones L, Tripp R, et al. Pattern recognition receptors TLR4 and CD14 mediate response to respiratory syncytial virus. Nat Immunol. 2000; 1(5): 398-401.

40. Smiley S, King J, Hancock W. Fibrinogen stimulates macrophage chemokine secretion through tolllike receptor 4. J Immunol. 2001; 167(5): 2887-94.

41. Termeer C, Benedix F, Sleeman J, Fieber C, Voith U, Ahrens T, et al. Oligosaccharides of hyaluronan activate dendritic cells via toll-like receptor 4. J Exp Med. 2002; 195(1): 99-111. 
42. Li F, Li X, Zou G, Gao Y, Ye J. Association between TLR7 copy number variations and hepatitis B virus infection outcome in Chinese (corrected: China). World J Gastroenterol. 2017; 23(9): 1602-7.

43. Horner S. Activation and evasion of antiviral innate immunity by hepatitis $C$ virus. J Mol Biol. 2014; 426(6): 1198-209.

44. Thimme R, Binder M, Bartenschlager R. Failure of innate and adaptive immune responses in controlling hepatitis $C$ virus infection. FEMS Microbiol Rev. 2012; 36(3): 663-83.

45. Nazmi A, Mukherjee S, Kundu K, Dutta K, Mahadevan A, Shankar S, et al. TLR7 is a key regulator of innate immunity against Japanese encephalitis virus infection. Neurobiol Dis. 2014; 69: 235-47.

46. Funk E, Kottilil S, Gilliam B, Talwani R. Tickling the TLR7 to cure viral hepatitis. J Transl Med. 2014; 12: 129.

47. Ou X, Mao S, Cao J, Cheng A, Wang M, Zhu D, et al. Comparative analysis of virus-host interactions caused by a virulent and an attenuated duck hepatitis A virus genotype 1. PLoS One. 2017; 12(6): e0178993.

48. Kanehisa M, Araki M, Goto S, Hattori M, Hirakawa M, Itoh M, et al. KEGG for linking genomes to life and the environment. Nucleic Acids Res. 2008; 36: 480-4.

49. Wu J, Mao X, Cai T, Luo J, Wei L. KOBAS server: a web-based platform for automated annotation and pathway identification. Nucleic Acids Res. 2006; 34: 720-4.

50. Wang X, Zhang J, Meng R, Jiang Y, Liang S, Zhang Y, et al. Host differences affecting resistance and susceptibility of the second generation of a Pekin duck flock to duck hepatitis A virus genotype 3 . Front. Microbiol. 2017; 8:

51. Cikos S, Bukovska A, Koppel J. Relative quantification of mRNA: comparison of methods currently used for real-time PCR data analysis. BMC Mol Biol. 2007; 8: 113.

\section{Tables}

Table 1A

\begin{tabular}{lcccc}
\hline Pathway & DEG in Pathway All Gene in Pathway & P-value & Q-value \\
\hline Influenza A & 55 & 134 & $1.58 \mathrm{E}-10$ & $3.48 \mathrm{E}-08$ \\
Cytokine-cytokine receptor interaction & 56 & 144 & $1.24 \mathrm{E}-09$ & $2.73 \mathrm{E}-07$ \\
Protein processing in endoplasmic reticulum & 66 & 187 & $4.65 \mathrm{E}-09$ & $1.02 \mathrm{E}-06$ \\
\hline Intestinal immune network for IgA production & 20 & 31 & $1.03 \mathrm{E}-08$ & $2.27 \mathrm{E}-06$ \\
Phagosome & 53 & 144 & $3.26 \mathrm{E}-08$ & $7.18 \mathrm{E}-06$ \\
Cell adhesion molecules (CAMs) & 44 & 111 & $3.98 \mathrm{E}-08$ & $8.76 \mathrm{E}-06$ \\
JAK-STAT signaling pathway & 44 & 120 & $5.58 \mathrm{E}-07$ & $0.000123 \mathbf{S}$ \\
Toll-like receptor signaling pathway & 29 & 83 & 0.0001322030 .029085 \\
\hline
\end{tabular}

Table 1B

\begin{tabular}{lccc}
\hline Pathway & DEG in Pathway All Gene in Pathway P-value & Q-value \\
\hline Cytokine-cytokine receptor interaction & 74 & 144 & $3.43 \mathrm{E}-08$ 8.44E-06 \\
Influenza A & 67 & 134 & $5.84 \mathrm{E}-070.000144$ \\
Toll-like receptor signaling pathway & 42 & 83 & $5.28 \mathrm{E}-050.012986$ \\
\hline
\end{tabular}


Table 1C

\begin{tabular}{lcccc}
\hline Pathway & $\begin{array}{l}\text { DEG in } \\
\text { Pathway }\end{array}$ & All Gene in Pathway & P-value & Q-value \\
\hline Glycine, serine and threonine & 17 & 52 & $6.74 \mathrm{E}-08$ & $9.37 \mathrm{E}-06$ \\
metabolism & 26 & 142 & $8.65 \mathrm{E}-06$ & 0.001202 \\
Neuroactive ligand-receptor interaction & 22 & 111 & $1.18 \mathrm{E}-05$ & 0.001637 \\
ECM-receptor interaction & 24 & 146 & 0.000118608 & 0.016487 \\
Calcium signaling pathway & 33 & 243 & 0.000311296 & 0.04327 \\
\hline Focal adhesion & & & &
\end{tabular}

Table 2

\begin{tabular}{|c|c|c|c|c|c|}
\hline \multirow[t]{2}{*}{ Gene } & \multirow[t]{2}{*}{ Abbreviation } & \multicolumn{2}{|c|}{$\begin{array}{l}\text { Transcriptomics fold } \\
\left.\text { change ( } \log _{2} \mathrm{FC}\right)\end{array}$} & \multicolumn{2}{|c|}{$\begin{array}{l}\text { Real-time RT-PCR fold } \\
\text { change }\left(2^{-\Delta \Delta C T}\right)\end{array}$} \\
\hline & & $\begin{array}{l}\text { DHV-1/ } \\
\text { control }\end{array}$ & $\begin{array}{l}\text { DHV-1a/ } \\
\text { control }\end{array}$ & $\begin{array}{l}\text { DHV-1/ } \\
\text { control }\end{array}$ & $\begin{array}{l}\text { DHV-1a/ } \\
\text { control }\end{array}$ \\
\hline Glycine N-methyltransferase-like & GNMT-like & -2.08 & -5.72 & 0.210 & 0.01 \\
\hline Glycine C-acetyltransferase & GCAT & -2.11 & -4.80 & 0.20 & 0.04 \\
\hline Cystathionine beta-synthase & CBS & -1.87 & -5.79 & 0.25 & 0.03 \\
\hline Phosphoglycerate dehydrogenase & PHGDH & -2.49 & -5.24 & 0.18 & 0.02 \\
\hline $\begin{array}{l}\text { Sarcoplasmic/endoplasmic reticulum } \\
\text { calcium ATPase }\end{array}$ & SERCA & 1.05 & 1.05 & 2.46 & 4.48 \\
\hline $\begin{array}{l}\text { 1-phosphatidylinositol 4,5- } \\
\text { bisphosphate phosphodiesterase gamma }\end{array}$ & PLC $\gamma$ & 1.58 & 1.37 & 4.96 & 2.95 \\
\hline Toll-like receptor 2 & TLR2 & 2.93 & 3.41 & 8.69 & 12.30 \\
\hline Toll-like receptor 4 & TLR4 & 5.27 & 5.69 & 38.05 & 46.85 \\
\hline Toll-like receptor 7 & TLR7 & 5.81 & 5.36 & 45.57 & 30.27 \\
\hline Interferon alpha & IFN $\alpha$ & 2.23 & 2.62 & 3.89 & 8.06 \\
\hline
\end{tabular}

Table 3

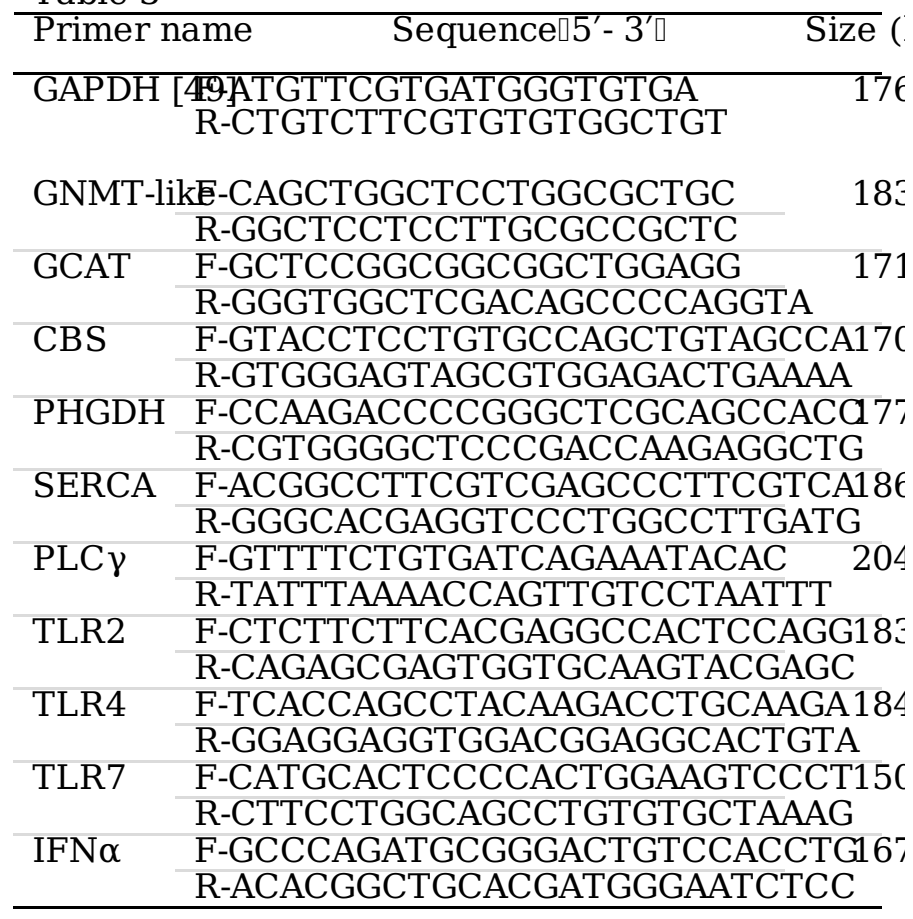
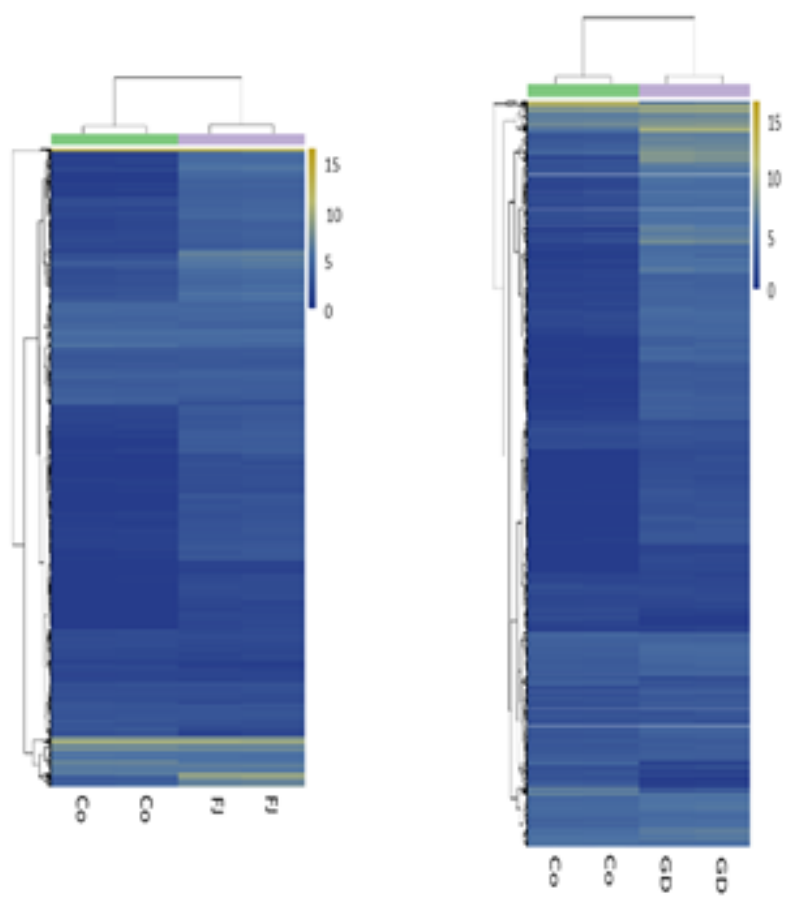

Figure 1

Heat map analysis used to classify gene expression patterns in DHAV-1-infection (A) and DHAV-1ainfection (B). Genes with similar expression patterns were clustered, as shown in the heat maps. Intensity of color indicated gene expression levels that were normalized according to Log2 FPKM value 

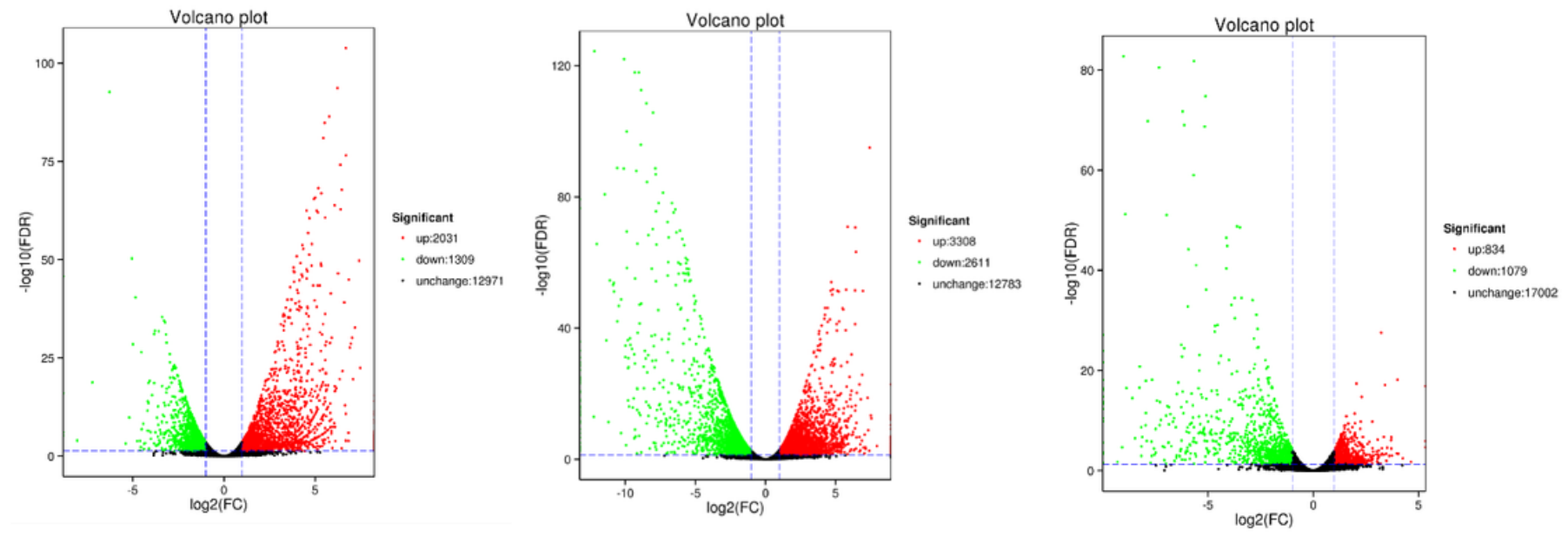

Figure 2

Volcano plots of differentially expressed genes in the pancreas of the ducklings in DHAV-1 vs control (A), DHAV-1a vs control (B) and DHAV-1a vs DHAV-1 (C). Red points represented up-regulated genes, green points represented down-regulated genes, and blue points represented genes with no significant difference
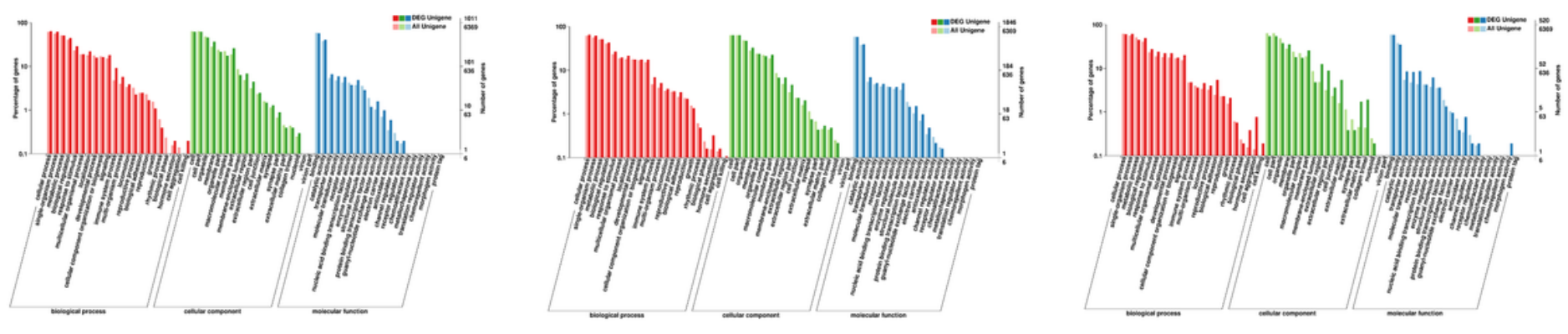

\section{Figure 3}

GO classification of differentially expressed genes in the pancreas of the ducklings in DHAV-1 vs control (A), DHAV-1a vs control (B) and DHAV-1a vs DHAV-1 (C). Annotation statistics of differentially expressed genes in the secondary node of GO. The horizontal axis showed secondary nodes of three categories in GO. The left side of vertical axis displayed the percentage of annotated genes versus the total gene number and the right side showed the number of genes 

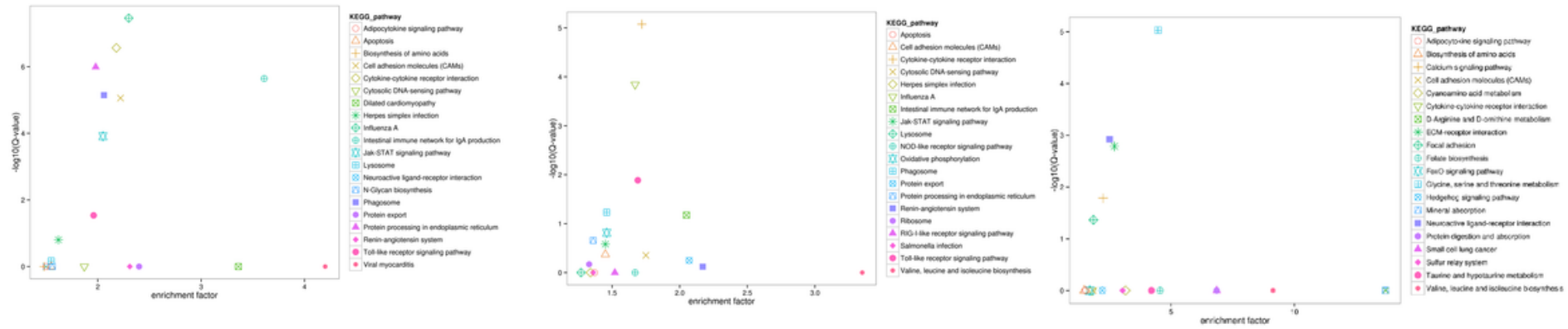

\section{Figure 4}

1. Kim M, Kim M, Kwon Y, Lindberg A, Joh S, Kwon H, et al. Development of duck hepatitis A virus type 3 vaccine and its use to protect ducklings against infections. Vaccine. 2009; 27(48): 6688-94. 2. Tseng C, Knowles N, Tsai H. Molecular analysis of duck hepatitis virus type 1 indicates that it should be assigned to a new genus. Virus Res. 2007; 123(2): 190-203. 3. Tseng C, Tsai H. Molecular characterization of a new serotype of duck hepatitis virus. Virus Res. 2007; 126(1-2): 19-31. 4. Fu Y, Pan M, Wang X, Xu Y, Yang H, Zhang D. Molecular detection and typing of duck hepatitis A virus directly from clinical specimens. Vet Microbiol. 2008; 131(3-4): 247-57. 5. Kim M, Kwon Y, Joh S, Kim S, Tolf C, Kim J, et al. Recent Korean isolates of duck hepatitis virus reveal the presence of a new geno- and serotype when compared to duck hepatitis virus type 1 type strains. Arch Virol. 2007; 152(11): 2059-72. 6. Liu M, Fanyi M, Li X, Zhang Z, Liu $S$, Zhang Y. Goose haemorrhagic hepatitis caused by a new subtype duck hepatitis type 1 virus. Vet Microbiol. 2011; 152(3-4): 280-3. 7. Wei C, Su S, Huang Z, Zhu W, Chen J, Zhao F, et al. Complete genome sequence of a novel duck hepatitis A virus discovered in southern China. J Virol. 2012; 86(18): 10247. 8. Guérin J, Noutary V, Boissieu C, Albaric O, Wyers M. Viral pancreatitis and encephalitis of Muscovy ducklings. Vet Rec. 2005; 157(11): 328. 9. Fu G, Chen H, Huang Y, Shi S. Identification and sequence analysis of duck hepatitis A virus type I isolated from Muscovy duckling with pancreatitis. FJ J Agri Sci. 2012; 27(9): 43-5. 10. Chen Z, Fu Q, Chen H, Fu G. Different pathogenicity of duck Hepatitis A virus 1 between pancreatitis-type and classical duckling. FJ J Agri Sci. 2013; 28 (10): 939-42. 11. Fu Q, Chen Z, Huang Y, Fu G. Identification of subtype of duck hepatitis A virus type 1 causing pancreatitis. Chin J Vet Med. 2015; 51(7): 36-8. 12. Schneider R, Shenk T. Impact of virus infection on host cell protein synthesis. Annu Rev Biochem. 1987; 56: 317-32. 13. Liu R, Shi S, Huang Y, Chen Z, Chen C, Cheng L, et al. Comparative pathogenicity of different subtypes of duck hepatitis A virus in Pekin ducklings. Vet Microbiol. 2019; 228: 181-7. 14. Zhu T, Qi B, Liu R, Jiang X, Lu R, Xiao L, et al. Comparative pathogenicity of two subtypes (hepatitis-type and pancreatitis-type) of duck hepatitis A virus type 1 in experimentally infected Muscovy ducklings. Avian Pathol. 2019; 48(4): 352-61. 15. Cha S, Roh J, Kang M, Kim B, Jang H. Isolation and characterization of a low pathogenic duck hepatitis A virus 3 from South Korea. Vet Microbiol. 2013; 162(1): 254-8. 16. Qian X, Ba Y, Zhuang Q, Zhong G. RNA-Seq technology and its application in fish transcriptomics. OMICS. 2014; 18(2): 98-110. 17. Wang Q, Liu M, Xu L, Wu Y, Huang Y. Transcriptome analysis reveals the molecular mechanism of hepatic fat metabolism disorder caused by Muscovy duck reovirus infection. Avian Pathol. 2018; 47(2): 127-39. 18. Wang X, Zhang J, Meng R, Jiang 
Y, Liang S, Zhang Y, et al. Host differences affecting resistance and susceptibility of the second generation of a Pekin duck flock to duck hepatitis A virus genotype 3. Front Microbiol. 2017; 8: 1128. 19. Yan L, Qu S, Liu G, Liu L, Yu Y, Ding G, et al. Comparative transcriptomic analysis of primary duck hepatocytes provides insight into differential susceptibility to DHBV infection. PLoS One. 2016; 11(2): e0149702. 20. Zhang X, Cao C, Liu Y, Qi H, Zhang W, Hao C, et al. Comparative liver transcriptome analysis in ducklings infected with duck hepatitis A virus 3 (DHAV-3) at 12 and 48 hours post-infection through RNA-seq. Vet Res. 2018; 49(1): 52. 21. Zhang Q. Research on the transcriptome of DHAV-1 infected duckling's livers through RNA-Seq. 2013, Huazhong Agricultural University: Wuhan. 22. Hart C, Race V, Achouri Y, Wiame E, Sharrard M, Olpin S, et al. Phosphoserine aminotransferase deficiency: a novel disorder of the serine biosynthesis pathway. Am J Hum Genet. 2007; 80(5): 931-7. 23. El-Hattab A. Serine biosynthesis and transport defects. Mol Genet Metab. 2016; 118(3): 153-9. 24. Zaki M, Thoenes M, Kawalia A, Nurnberg P, Kaiser R, Heller R, et al. Recurrent and prolonged infections in a child with a homozygous IFIH1 nonsense mutation. Front Genet. 2017; 8: 130. 25. Jiang H, Yu Y, Liu S, Zhu M, Dong $\mathrm{X}, \mathrm{Wu} \mathrm{J}$, et al. Proteomic study of a Parkinson's disease model of undifferentiated SH-SY5Y cells induced by a proteasome inhibitor. Int J Med Sci. 2019; 16(1): 84-92. 26. Sim W, Yin H, Choi H, Choi Y, Kwak H, Kim $\mathrm{S}$, et al. L-serine supplementation attenuates alcoholic fatty liver by enhancing homocysteine metabolism in mice and rats. J Nutr. 2015; 145(2): 260-7. 27. Sun L, Bartlam M, Liu Y, Pang H, Rao Z. Crystal structure of the pyridoxal-5'-phosphate-dependent serine dehydratase from human liver. Protein Sci. 2005; 14(3): 791-8. 28. Talukdar G, Inoue R, Yoshida T, Ishimoto T, Yaku K, Nakagawa T, et al. Novel role of serine racemase in anti-apoptosis and metabolism. Biochim Biophys Acta Gen Subj. 2017; 1861(1): 3378-87. 29. Ogawa H, Gomi T, Fujioka M. Serine hydroxymethyltransferase and threonine aldolase: are they identical? Int J Biochem Cell Biol. 2000; 32(3): 289-301. 30. Cario E. Bacterial interactions with cells of the intestinal mucosa: Toll-like receptors and NOD2. Gut. 2005; 54(8): 1182-93. 31. Takeuchi O, Akira S. Innate immunity to virus infection. Immunol Rev. 2009; 227(1): 75-86. 32. Aderem A, Ulevitch R. Toll-like receptors in the induction of the innate immune response. Nature. 2000; 406(6797): 782-7. 33. Medzhitov R, Preston-Hurlburt P, Janeway C. A human homologue of the Drosophila Toll protein signals activation of adaptive immunity. Nature. 1997; 388(6640): 394-7. 34. Dolganiuc A, Oak S, Kodys K, Golenbock D, Finberg R, Kurt-Jones $\mathrm{E}$, et al. Hepatitis $\mathrm{C}$ core and nonstructural 3 proteins trigger toll-like receptor 2mediated pathways and inflammatory activation. Gastroenterology. 2004; 127(5): 1513-24. 35. Feldmann G, Nischalke H, Nattermann J, Banas B, Berg T, Teschendorf C, et al. Induction of interleukin- 6 by hepatitis $\mathrm{C}$ virus core protein in hepatitis $\mathrm{C}$-associated mixed cryoglobulinemia and $\mathrm{B}$-cell non-Hodgkin's lymphoma. Clin Cancer Res. 2006; 12(15): 4491-8. 36. Arbour N, Lorenz E, Schutte B, Zabner J, Kline J, Jones $\mathrm{M}$, et al. TLR4 mutations are associated with endotoxin hyporesponsiveness in humans. Nat Genet. 2000; 25(2): 187-91. 37. Lorenz E, Mira J, Frees K, Schwartz D. Relevance of mutations in the TLR4 receptor in patients with gram-negative septic shock. Arch Intern Med. 2002; 162(9): 1028-32. 38. Qureshi S, Lariviere L, Leveque G, Clermont S, Moore K, Gros P, et al. Endotoxin-tolerant mice have mutations in Toll-like receptor 4 (TIr4). J Exp Med. 1999; 189(4): 615-25. 39. Kurt-Jones E, Popova L, Kwinn L, Haynes $L$, Jones L, Tripp R, et al. Pattern recognition receptors TLR4 and CD14 mediate response to respiratory syncytial virus. Nat Immunol. 2000; 1(5): 398-401. 40. Smiley S, King J, Hancock W. Fibrinogen stimulates macrophage chemokine secretion through toll-like receptor 4. J Immunol. 2001; 167(5): 2887-94. 41. 
Termeer C, Benedix F, Sleeman J, Fieber C, Voith U, Ahrens T, et al. Oligosaccharides of hyaluronan activate dendritic cells via toll-like receptor 4. J Exp Med. 2002; 195(1): 99-111. 42. Li F, Li X, Zou G, Gao Y, Ye J. Association between TLR7 copy number variations and hepatitis B virus infection outcome in Chinese (corrected: China). World J Gastroenterol. 2017; 23(9): 1602-7. 43. Horner S. Activation and evasion of antiviral innate immunity by hepatitis $C$ virus. J Mol Biol. 2014; 426(6): 1198-209. 44. Thimme $\mathrm{R}$, Binder $\mathrm{M}, \mathrm{Bartenschlager} \mathrm{R}$. Failure of innate and adaptive immune responses in controlling hepatitis $\mathrm{C}$ virus infection. FEMS Microbiol Rev. 2012; 36(3): 663-83. 45. Nazmi A, Mukherjee S, Kundu K, Dutta K, Mahadevan A, Shankar S, et al. TLR7 is a key regulator of innate immunity against Japanese encephalitis virus infection. Neurobiol Dis. 2014; 69: 235-47. 46. Funk E, Kottilil S, Gilliam B, Talwani R. Tickling the TLR7 to cure viral hepatitis. J TransI Med. 2014; 12: 129. 47. Ou X, Mao S, Cao J, Cheng A, Wang M, Zhu $D$, et al. Comparative analysis of virus-host interactions caused by a virulent and an attenuated duck hepatitis A virus genotype 1. PLoS One. 2017; 12(6): e0178993. 48. Kanehisa M, Araki M, Goto S, Hattori $M$, Hirakawa $M$, Itoh $M$, et al. KEGG for linking genomes to life and the environment. Nucleic Acids Res. 2008; 36: 480-4. 49. Wu J, Mao X, Cai T, Luo J, Wei L. KOBAS server: a web-based platform for automated annotation and pathway identification. Nucleic Acids Res. 2006; 34: 720-4. 50. Wang X, Zhang J, Meng R, Jiang Y, Liang S, Zhang Y, et al. Host differences affecting resistance and susceptibility of the second generation of a Pekin duck flock to duck hepatitis A virus genotype 3. Front. Microbiol. 2017; 8: 1128. 51. Cikos S, Bukovska A, Koppel J. Relative quantification of mRNA: comparison of methods currently used for real-time PCR data analysis. BMC Mol Biol. 2007; 8: 113. Table and figure legends Table 1 Signal pathway enrichment evaluated by KEGG in the pancreas of the ducklings in DHAV-1 vs control (A), DHAV1 a vs control (B) and DHAV-1a vs DHAV-1 (C) Table 2 Verifcation of real-time RT-PCR for some diferentially expressed genes Table 3 Primer sequences of differentially expressed genes for real-time RTPCR analysis Figure 1 Heat map analysis used to classify gene expression patterns in DHAV-1-infection (A) and DHAV-1a-infection (B). Genes with similar expression patterns were clustered, as shown in the heat maps. Intensity of color indicated gene expression levels that were normalized according to Log2 FPKM value Figure 2 Volcano plots of differentially expressed genes in the pancreas of the ducklings in DHAV-1 vs control (A), DHAV-1a vs control (B) and DHAV-1a vs DHAV-1 (C). Red points represented upregulated genes, green points represented down-regulated genes, and blue points represented genes with no significant difference Figure $3 \mathrm{GO}$ classification of differentially expressed genes in the pancreas of the ducklings in DHAV-1 vs control (A), DHAV-1a vs control (B) and DHAV-1a vs DHAV-1 (C). Annotation statistics of differentially expressed genes in the secondary node of GO. The horizontal axis showed secondary nodes of three categories in $\mathrm{GO}$. The left side of vertical axis displayed the percentage of annotated genes versus the total gene number and the right side showed the number of genes Figure 4 Top 20 KEGG pathways enriched in the pancreas of the ducklings in DHAV-1 vs control (A), DHAV-1a vs control (B) and DHAV-1a vs DHAV-1 (C)

\section{Supplementary Files}

This is a list of supplementary files associated with this preprint. Click to download. 
- Supplementallnformation.docx 